\title{
Nonlinear dynamics of electromagnetic pulses in cold relativistic plasmas
}

\author{
A. B ONATTO, R. PAKTER and F. B. R IZZATO \\ Instituto de Física, Universidade Federal do Rio Grande do Sul, Caixa Postal 15051, \\ 91501-970 Porto Alegre, Rio Grande do Sul, Brazil
}

(Received 30 October 2004, in revised form 8 April 2005 and accepted 12 April 2005)

\begin{abstract}
In the present analysis we study the self-consistent propagation of nonlinear electromagnetic pulses in a one-dimensional relativistic electron-ion plasma, from the perspective of nonlinear dynamics. We show how a series of Hamiltonian bifurcations give rise to the electric fields which are of relevance in the subject of particle acceleration. Connections between these bifurcated solutions and the results of earlier analysis are made.
\end{abstract}

\section{Introduction}

The propagation of intense electromagnetic pulses in plasmas is a subject of current interest in a variety of areas that make use of the available modern laser technologies, among which we include particle and photon acceleration, nonlinear optics, laser fusion and others (Tajima and Dawson 1979; Shukla et al. 1986; Mendonça 2001; Poornakala et al. 2002; Bingham 2003). Intense electromagnetic pulses displace plasma electrons and create a resulting ambipolar electric field with the associated density fields. Under appropriate conditions all fields act coherently and the pulse keeps it shape. Studies on pulse localization have been performed in a variety of forms to unravel the corresponding numerical and analytical properties. Kozlov et al. (1979) investigated numerically the propagation of coupled electromagnetic and electrostatic modes in cold relativistic electron-ion plasmas and concluded that small- and large-amplitude localized solutions can be present. Mofiz and de Angelis (1985) applied analytical approximations to the same model and suggested where and how localized solutions can be obtained. Ensuing, more recent works provide an even deeper understanding as various features have been investigated, such as the influence of ion motion in slow, ion-accelerating solitons (Farina and Bulanov 2001), the existence of moving solitons (Poornakala et al. 2002), the existence of trails lagging isolated pulses (Kuehl and Zang 1993; Sudan et al. 1997) and others. Some key points, however, remain not quite understood, such as the way small-amplitude localized solutions are destroyed; when isolated pulses are actually free of smaller-amplitude trails; and more specific properties of the spectrum of stronger-amplitude solutions, to mention a few. Those are issues of relevance if one wishes to establish the existence range and stability properties of the localized modes.

In the present paper we shall turn our attention to small-amplitude solitons propagating in underdense rarified plasmas, since this kind of soliton may be of relevance for wakefield schemes. In doing so we shall follow an alternative 
strategy, other than the direct integration of the governing equations which has been the standard approach so far. We intend to examine the problem using the techniques of nonlinear dynamics (Rizzato et al. 1997). A canonical representation shall be constructed in association with several tools from nonlinear dynamics such as Poincaré maps and stability matrices. This strategy naturally provides a clear way to investigate the system since we intend to establish a connection between the pulses of radiation and fixed points of the corresponding nonlinear dynamical system (Lichtenberg and Lieberman 1992). Several facts are known and we state some which are of direct relevance for our analysis: small-amplitude solitons are created as the wave system becomes modulationally unstable at an upper limit of the carrier frequency and cease to exist beyond the lower limit of this carrier frequency. Not much is known as to how solitons are destroyed at the lower boundary and we examine this point to show that a series of nonlinear resonances and bifurcations are responsible for the process. A related relevant problem is when isolated pulses are actually free of smaller-amplitude trails and this has to do with the existence of wakefields following the leading wave front which is of relevance for particle acceleration, for instance. Those are basic issues if one wishes to operate the wave system under conditions suited for particle acceleration, and our purpose with the present paper is to contribute towards the analysis of these aspects.

\section{The model}

We follow previous works and model our system as consisting of two cold relativistic fluids: one electronic, the other ionic. Electromagnetic radiation propagates along the $z$ axis of our coordinate system and we represent the relevant fields in the dimensionless forms $e \mathbf{A}(z, t) / m_{\mathrm{e}} c^{2} \rightarrow \mathbf{A}(z, t)$ for the laser vector potential and $e \phi(z, t) / m_{\mathrm{e}} c^{2} \rightarrow \phi(z, t)$ for the electric potential. $-e$ is the electron charge, $m_{\mathrm{e}}$ is its mass and $c$ is the speed of light; $m_{\mathrm{i}}$ will denote ionic mass when appropriate. In addition, we suppose stationary modulations of a circularly polarized carrier wave for the vector potential in the form $\mathbf{A}(z, t)=\psi(\tilde{\xi})[\hat{\mathbf{x}} \sin (k z-\omega t)+\hat{\mathbf{y}} \cos (k z-\omega t)]$ with $\tilde{\xi} \equiv z-V t$, whereupon introducing the expression for the vector potential into the governing Maxwell's equation one readily obtains $V=c^{2} k / \omega . V$ could be thus read as a nonlinear group velocity since we shall be working in regimes where $\omega$ and $k$ are related by a nonlinear dispersion relation. Manipulation of the governing equations finally takes us to the point where two coupled equations must be integrated - one controlling the vector potential and the other the electric potential (Kozlov et al. 1979; Mofiz and de Angelis 1985):

$$
\begin{aligned}
\psi^{\prime \prime} & =-\frac{1}{\eta} \psi+\frac{V_{0}}{p} \psi\left[\frac{1}{r_{\mathrm{e}}(\phi, \psi)}+\frac{\mu}{r_{\mathrm{i}}(\phi, \psi)}\right], \\
\phi^{\prime \prime} & =\frac{V_{0}}{p}\left[\frac{(1+\phi)}{r_{\mathrm{e}}(\phi, \psi)}-\frac{(1-\mu \phi)}{r_{\mathrm{i}}(\phi, \psi)}\right],
\end{aligned}
$$

where the primes denote derivatives with respect to $\xi \equiv\left(\omega_{\mathrm{e}} / c\right) \tilde{\xi}, r_{\mathrm{e}}(\phi, \psi) \equiv$ $\sqrt{(1+\phi)^{2}-p\left(1+\psi^{2}\right)}, r_{\mathrm{i}}(\phi, \psi) \equiv \sqrt{(1-\mu \phi)^{2}-p\left(1+\mu^{2} \psi^{2}\right)}, \eta \equiv \omega_{\mathrm{e}}^{2} / \omega^{2}, \mu \equiv$ $m_{\mathrm{e}} / m_{\mathrm{i}}, V_{0} \equiv V / c$ and $p \equiv 1-V_{0}^{2}$, with $\omega_{\mathrm{e}}^{2} \equiv 4 \pi n_{\mathrm{e}} e^{2} / m_{\mathrm{e}}$ as the plasma frequency and $n_{\mathrm{e}}=n_{\mathrm{i}}$ as the equilibrium densities. We further rescale $\omega / c k \rightarrow \omega$ and $\omega_{\mathrm{e}} / c k \rightarrow \omega_{\mathrm{e}}$ in $V_{0}, \eta$ and $p$, which helps to simplify the coming investigation: $\eta$ preserves its form, $V_{0} \rightarrow 1 / \omega$ and $p \rightarrow 1-1 / \omega^{2}$. A noticeable feature of the system (2.1) and (2.2) is 
that it can be written as a Hamiltonian system of a quasi-particle with two degrees of freedom. Indeed, if one introduces the momenta $P_{\psi} \equiv \psi^{\prime}$ and $P_{\phi} \equiv-\phi^{\prime} / p$, the equations for $\psi$ and $\phi$ take the form

$$
\begin{aligned}
& \psi^{\prime}=\partial H / \partial P_{\psi}, \quad P_{\psi}^{\prime}=-\partial H / \partial \psi, \\
& \phi^{\prime}=\partial H / \partial P_{\phi}, \quad P_{\phi}^{\prime}=-\partial H / \partial \phi
\end{aligned}
$$

where the Hamiltonian $H$ reads as

$$
H=\frac{P_{\psi}^{2}}{2}-p \frac{P_{\phi}^{2}}{2}+\frac{1}{2 \eta} \psi^{2}+\frac{V_{0}}{p^{2}}\left[r_{\mathrm{e}}(\phi, \psi)+\frac{1}{\mu} r_{\mathrm{i}}(\phi, \psi)\right]
$$

$H$ is constant since it does not depend on the 'time' variable $\xi$. Its constant value, let us call it $E$, can be calculated as soon as the appropriate initial conditions are specified. In our case we shall be interested in the propagation of pulses vanishing for $|\xi| \rightarrow \infty$, so we know that the conditions $P_{\psi}=P_{\phi}=\phi=\psi=0$ must pertain to the relevant dynamics, from which one concludes that $E=\left(V_{0} / p\right)^{2}(1+1 / \mu)$. Additional conditions arise from the presence of square roots in the Hamiltonian; the dynamics lies within regions where simultaneously $r_{\mathrm{e}}^{2}, r_{\mathrm{i}}^{2}>0$. Combining these inequalities with the boundary conditions one is led to conclude that the entire dynamics must evolve within the physical region

$$
\phi_{\min } \equiv \sqrt{p\left(1+\psi^{2}\right)}-1<\phi<\frac{1}{\mu}\left[1-\sqrt{p\left(1+\mu^{2} \psi^{2}\right)}\right] \equiv \phi_{\max }
$$

if $p>0$. If $p<0$ there is no restriction, but we shall see that only positive values of $p$ are of interest here. We can also evaluate the linear frequencies of laser and wakefield small fluctuations in the form

$$
\psi^{\prime \prime}=\Omega_{\psi}^{2} \psi, \phi^{\prime \prime}=-\Omega_{\phi}^{2} \phi,
$$

where

$$
\Omega_{\psi}^{2} \equiv-1 / \eta+1 / p(1+\mu) \text { and } \Omega_{\phi}^{2} \equiv(1+\mu) / V_{0}^{2} .
$$

The potential $\phi$ oscillates with a real frequency $\Omega_{\phi}$ which can be shown to convert into $\omega_{\mathrm{e}}(1+\mu)^{1 / 2}$ if dimensional variables are used for space and time. As for the vector potential, to reach high-intensity fields from noise level radiation, instability must be present, which demands $\Omega_{\psi}^{2}>0$ and, consequently from relation (2.8),

$$
1<\omega^{2} \leqslant 1+\omega_{\mathrm{e}}^{2}(1+\mu),
$$

so $p>0$.

The threshold $\Omega_{\psi}^{2}=0$ can be rewritten in the form $\omega=\omega_{*} \equiv \sqrt{1+\omega_{\mathrm{e}}^{2}(1+\mu)}$, where $\omega_{*}$ is the linear dispersion relation for electromagnetic waves. What we expect to see are small-amplitude waves when $\omega$ is slightly smaller than $\omega_{*}$, with amplitudes increasing as we move farther from the threshold. In addition to that, another feature worthy of notice must be commented on. If one sits very close to the threshold, amplitude modulations of the laser field are tremendously slow, while the oscillatory frequency of the electric potential $\phi$ remains relatively high. The resulting frequency disparity provides the conditions for a slow adiabatic dynamics where, given a slowly varying $\psi, \phi$ always accommodates itself close to the minimum of

$$
U(\phi, \psi) \equiv-V_{0} / p^{2}\left[r_{\mathrm{e}}(\phi, \psi)+\mu^{-1} r_{\mathrm{i}}(\phi, \psi)\right],
$$




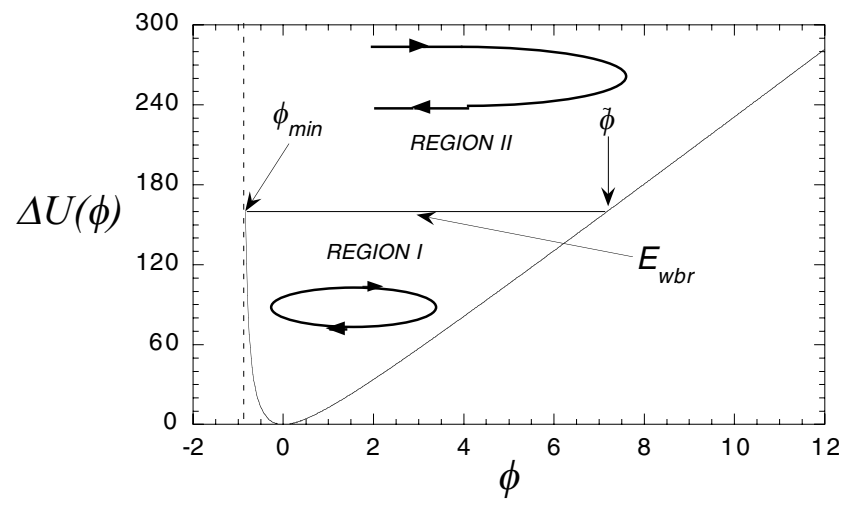

Figure 1. Oscillating $(I)$ and wave breaking $(I I)$ regions for the electric potential at $\psi=0 . \Delta U$ is defined in the text.

with the 'minus' sign on the right-hand side accounting for the negative effective mass of $\phi$ as seen in (2.5); note that $\phi_{\min }$ of (2.6) refers to the smallest available $\phi$ and not to the minimum of $U$. When $\psi=0$, a condition to be used shortly in our Poincaré plots, $U$ has a minimum at $\phi=0$ which is thus a stable point in the adiabatic regime. As one moves away from the threshold, faster modulations and higher amplitudes may be expected to introduce considerable amounts of nonintegrable behavior and chaos into the system. This kind of perspective agrees well with the result of previous works where adiabatic regions have been interpreted to be essentially associated with small-amplitude quasi-neutral dynamics (Kozlov et al. 1979). One of our interests here is to see precisely how the adiabatic dynamics is broken as one moves deeper into non-integrable regimes. An additional fact must be observed as one searches for adiabatic solutions and this has to do with how close to the minimum of $U$ one must sit to find these adiabatic solutions. The corresponding discussion parallels that on wave breaking of relativistic electrostatic waves. First of all note that if we do not set $\phi$ right at the respective minimum of $U$, the electric potential will oscillate around the minimum which will be itself displaced due to the action of the slowly varying $\psi$. Again when $\psi=0$, inequality (2.6) reveals that $\phi$ must lie in the range $\phi_{\min }=\sqrt{p}-1<0$ to $\phi_{\max }=1 / \mu(1-\sqrt{p})>0$. Not all of these values are, however, actually allowed in adiabatic dynamics. Oscillations will occur consistently only if the orbit is free to wander to the right- and left-hand sides of the minimum $\phi=0$ and this can only happen when the oscillating orbit is entirely trapped within the attracting well of $U . U<0$, and a quick calculation shows that

$$
U\left(\phi_{\min }\right)^{2}-U\left(\phi_{\max }\right)^{2}=2 \sqrt{p}\left(1-\mu^{2}\right)(1-\sqrt{p}) V_{0}^{2} \mu^{-2} p^{-7 / 2}>0,
$$

so $U\left(\phi_{\max }\right)>U\left(\phi_{\min }\right)$, which sets a limit to cyclic orbits: $\phi$ must be such that the corresponding potential will never be above the level $U\left(\phi_{\min }\right)$. To illustrate all of these comments, the reader is referred to Fig. 1 where the potential $\Delta U \equiv U(\phi, \psi=$ $0)-U(\phi=0, \psi=0)$ is represented for $V_{0}=0.99$ and $\mu=0.0005$, parameters characterizing high-velocity pulses with $U\left(\phi_{\tilde{\max }}\right) \gg U\left(\phi_{\min }\right)$ to be further investigated in Sec. 3: orbits of region $I, \phi_{\min }<\phi<\tilde{\phi}$, will oscillate back and forth, but orbits in region $I I$ eventually reach $\phi_{\min }$ where $r_{\mathrm{e}} \rightarrow 0$. Since it can be shown that the 
electronic density depends on $r_{\mathrm{e}}$ in the form $n_{\mathrm{e}} \sim r_{\mathrm{e}}^{-1}$ (Kozlov et al. 1979; Mofiz and de Angelis 1985), break down of the theory indicates wave breaking on electrons.

We point out that wave breaking occurs on electrons solely when the group velocity of the wave is close to the speed of light, which is the case of Fig. 1. At lower velocities $V_{0} \rightarrow 0$, from (2.11) $U\left(\phi_{\min }\right)^{2} \sim U\left(\phi_{\max }\right)^{2}$. This levels the borders of left- and right-hand side branches of the potential $U$, and consequently wave breaking can also occur on ions.

Also shown in the figure is the wave breaking energy

$$
E_{\mathrm{wbr}} \equiv \Delta U\left(\phi_{\min }\right)=\frac{V_{0}^{2}}{p^{2}}\left[1+\frac{1}{\mu}-\frac{1}{\mu V_{0}} \sqrt{\left(1-\mu \phi_{\min }\right)^{2}-p}\right] \approx \frac{\omega^{3}}{\omega_{\mathrm{e}}^{3}} \quad \text { if } \mu, p \ll 1,
$$

separating regions $I$ and $I I$. Our conclusion is that even with extremely slow modulations, oscillations of $\phi$ must be limited so as to satisfy the conditions discussed above. Not only that, but the very same figure suggests how non-integrability affects localization of our solutions: as one moves away from adiabaticity and into chaotic regimes, trajectories initially trapped by $U$ may be expected to chaotically diffuse towards upper levels of this effective potential, escaping from the trapping region, approaching $E_{\mathrm{wbr}}$ and eventually hitting the boundary at $\phi_{\min }$ or, in general, attaining $r_{\mathrm{e}}=0$ for $\psi \neq 0$. If this is so, we have an explanation of how smallamplitude solitons are destroyed, one of the issues of interest in the subject area (Poornakala et al. 2002). We now look at the problem with the help of methods of nonlinear dynamics.

\section{Analysis with nonlinear dynamics}

We introduce our Hamiltonian phase space in the form of a Ponicaré surface of section mapping where the pair of variables $\left(\phi, P_{\phi}\right)$ is recorded each time the plane $\psi=0$ is punctured with $P_{\psi}<0$. Once we have defined the map in this way, we can also investigate the existence and stability of periodic solutions of our coupled set of equations with the aid of a Newton-Raphson algorithm. The Newton-Raphson method locates periodic orbits and evaluates the corresponding stability index $\alpha$ which satisfies $|\alpha|<(>) 1$ for stable (unstable) trajectories (Pakter and Rizzato 2001). Parameters are represented in a form already used in earlier investigations on the subject: we first set a numerical value for $V_{0}$ and then obtain $\omega=1 / V_{0}$, which must be larger than unity as demanded by condition (2.9). However, we shall keep $V_{0}$ close to unity, and thus $\omega$ slightly larger than unity, so as to represent wave modes propagating at nearly the speed of light. This is the convenient setting if one is interested in fast electron acceleration by wakefields. After $V_{0}$ is established, the electron plasma frequency is calculated as $\omega_{\mathrm{e}}^{2}=\eta \omega^{2}$, with $\eta$ satisfying condition (2.9) again. We note that $\eta=\omega_{\mathrm{e}}^{2} / \omega^{2}=V_{0}^{2} \omega_{\mathrm{e}}^{2}=V_{0}^{2} \omega_{\mathrm{e}, \text { non-scaled }}^{2} / c^{2} k^{2}$; so holding $V_{0}$ constant while increasing $\eta$, is entirely equivalent to the more usual practice of holding $V_{0}$ and the original $\omega_{\mathrm{e}}$ constant, while decreasing $k$ and the original $\omega$. In all cases analyzed here we take $\mu=0.0005$ as in Kozlov et al. (1979). In addition to that, we shall take $V_{0}=0.99$ to represent the high-speed conditions of wakefield schemes. Now a crucial step is this: since isolated pulses cannot be seen in periodic plots we alter the energy $E$ slightly to $E=V_{0} / p^{2}(1+1 / \mu)(1+\epsilon), \epsilon \ll 1$ so the vanishing tail $P_{\psi}=P_{\phi}=\psi=\phi=0$ is avoided. With this maneuver we convert isolated pulses into trains of quasi-isolated pulses, a situation amenable to the use of nonlinear dynamics and the associated periodic plots; periodicity is, in fact, 
physically meaningful if pulses result from periodic self-modulations of initially uniform modes (Joshi and Katsouleas 2003). In all cases we make sure that as $\epsilon \rightarrow 0$ the trains go into individual packets and convergence is attained. The instability threshold for the vector potential is obtained in the form $\eta_{*}=p /(1+\mu)=0.0198$ so $\omega_{\mathrm{e}} \ll \omega$, as it must be in the underdense plasmas. To investigate the adiabatic regime of the relevant nonlinear dynamics we examine phase portraits for $\eta$ slightly larger than $\eta_{*}$. In Fig. 2(a) we set $\eta=1.00001 \eta_{*}$. With such a relatively small departure from marginal stability, modulations are slow with $\left|\Omega_{\phi}\right| \gg\left|\Omega_{\psi}\right|$, adiabatic approximations are thus fully operative and what we see in phase space is just a set of concentric Kolomogrov-Arnold-Moser (KAM) surfaces, rendering the system nearly integrable. The central fixed point corresponds to an isolated periodic orbit since it represent a phase-locked solution that returns periodically to $\psi=0$, $\phi \rightarrow 0$, and the surrounding curves depict regimes of quasi-periodic, non-vanishing fluctuations of $\phi$. Resonant islands are already present but still do not affect the central region of the phase plot where the solitary solution resides. In general, we have observed that increasingly large resonance islands are present away from the central region. When $\eta$ grows the behavior of the central fixed point can be observed in terms of its stability index represented in Fig. 2(b). The index oscillates within the stable range initially, which marks the existence of a central elliptic point near the origin. The stability index however finally reaches $\alpha=+1$ as indicated in the figure, beyond which point no central orbit is found. This indicates a tangent bifurcation with a neighboring orbit which terminates the existence of the central point (Rizzato and Pakter 2002). Immediately after tangency, the phase plot at $\psi=0$ is still constricted to small values of $\phi$ as can be seen in Fig. 2(c) where $\eta=1.0001 \eta_{*}$. Larger values of $\eta$ cause diffusion towards upper levels of $U(\phi)$ and we can see that in Fig. 3, where we investigate the behavior of the energy

$$
E_{\phi} \equiv p P_{\phi}^{2} / 2+\Delta U
$$

corresponding to the electrostatic field $\phi$. Instead of working directly with the form (3.1) we represent diffusion in terms of compact variables

$$
\begin{gathered}
e_{\phi} \equiv \frac{\chi_{\mathrm{e}} E_{\phi}}{\chi_{\mathrm{e}}+E_{\phi}}, \\
\Phi \equiv \frac{\chi_{\phi} \phi}{\chi_{\phi}+|\phi|},
\end{gathered}
$$

where $\chi_{\mathrm{e}}$ and $\chi_{\phi}$ represent the scale above which the corresponding variables are compacted. This kind of choice allows us to represent in the same plot the very extensive variations of energy and electric potential, without deforming these quantities when they are small, near their initial conditions. We found it convenient to use $\chi_{\mathrm{e}}=\chi_{\phi}=0.0001$ to discuss diffusion. In Fig. 3(a) we take $\eta=1.00001 \eta_{*}$ so we are in the regular regime; as expected, no diffusion is observed and the quasi-particle stays near its initial condition $P_{\phi}=0, \phi=10^{-8}$. For $\eta=1.00021 \eta_{*}$ as in panel (b), the central fixed point no longer exist. In addition to that, KAM surfaces no longer isolate the central region of the phase plot and diffusion is observed. The quasiparticle moves toward $E_{\mathrm{wbr}}$ and eventually arrives at this critical energy producing wave breaking on electrons. At this point the simulation stops with the electron density diverging to infinity. Diffusion is initially slow and becomes faster as energy increases. One sees voids in the diffusion plots which correspond to resonant islands 

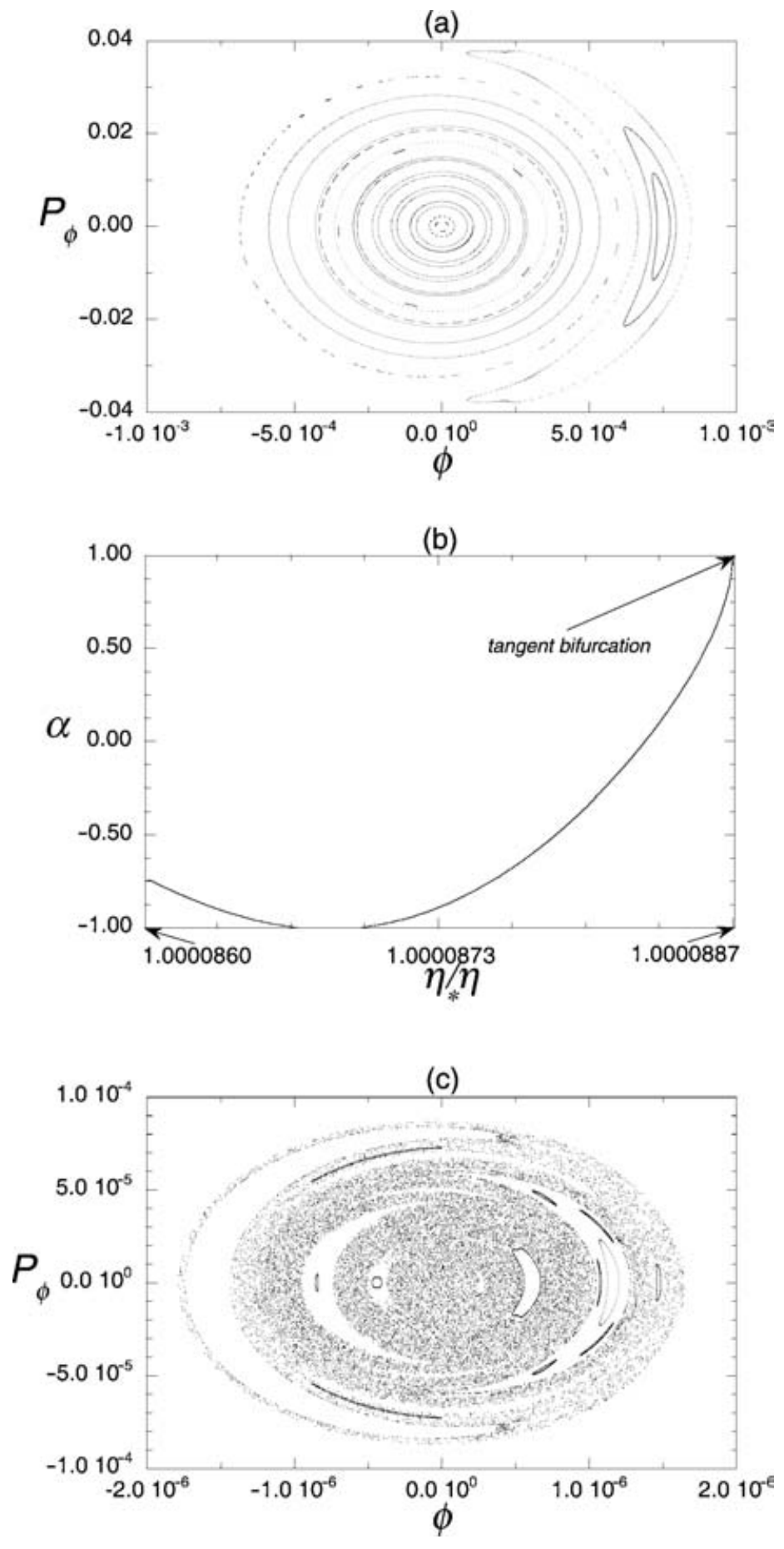

Figure 2. (a) Phase plot near the modulational instability threshold, with $\eta=1.00001 \eta_{*}$; (b) stability index versus $\eta / \eta_{*}$; (c) phase plot after the inverse tangency seen in (b), with $\eta=1.0001 \eta_{*} . \epsilon=10^{-11}$. 
(a)

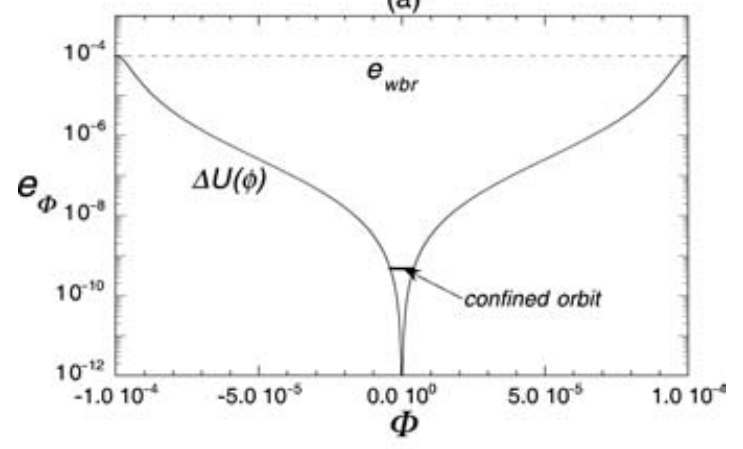

(b)

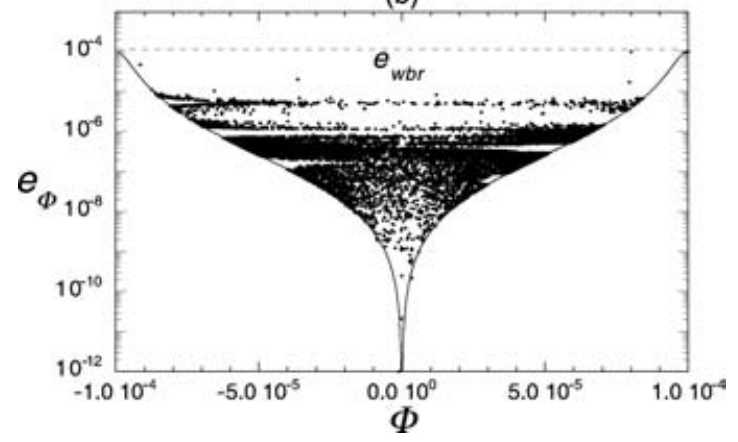

Figure 3. Dynamics as represented in the $e_{\phi}$ versus $\Phi$ space: (a) $\eta=1.00001 \eta_{*}$; (b) $\eta=1.00021 \eta_{*} . e_{\mathrm{wbr}} \equiv \chi_{\mathrm{e}} E_{\mathrm{wbr}} /\left(\chi_{\mathrm{e}}+E_{\mathrm{wbr}}\right)$.

in the phase space, so as diffusion proceeds the quasi-particle escalates along the contours of the resonances that become progressively larger as already mentioned this is why the process is initially slow, becoming faster in the final stages. For larger values of $\eta$ no resonance is present and the quasi-particle moves quickly toward $E_{\text {wbr }}$. In case of panel (b) one can still see various pulses before wave breaking, but when $\eta$ is so large that resonances are no longer present, wave breaking can be instantaneous. We finally note the following relevant fact. For $V_{0} \rightarrow 1$, it is known that the amplitude of the electromagnetic pulses are small (Poornakala et al. 2002). But as one goes beyond the adiabatic regime, our discussion on diffusion allows us to conclude that even small initial pulses eventually reach very high-amplitude values for the plasma waves, which provides the condition for formation of strong electric fields with the corresponding implications on particle acceleration. We illustrate the feature with a final figure, Fig. 4 , where, in a diffusive regime with $\eta=1.0004 \eta_{*}$, the electric field $-\phi^{\prime}=p P_{\phi}$ is shown to evolve from small values near initial conditions to the limiting wave breaking value which agrees with the calculated value - from (2.12) and Fig. $1-\left|\phi^{\prime}\right| \sim \sqrt{2 \omega / \omega_{\mathrm{e}}} \sim 3.5$.

We read all of these features as follows. For small enough $\eta$ there are locked solutions representing isolated pulses coexisting with surrounding quasi-periodic solutions where $\phi$ does not quite vanish when $\psi$ does. As $\eta$ increases past the mentioned tangent bifurcation but prior to full destruction of isolating KAM surfaces, one reaches a regime of periodical returns to $\psi=0$, although in the presence of 


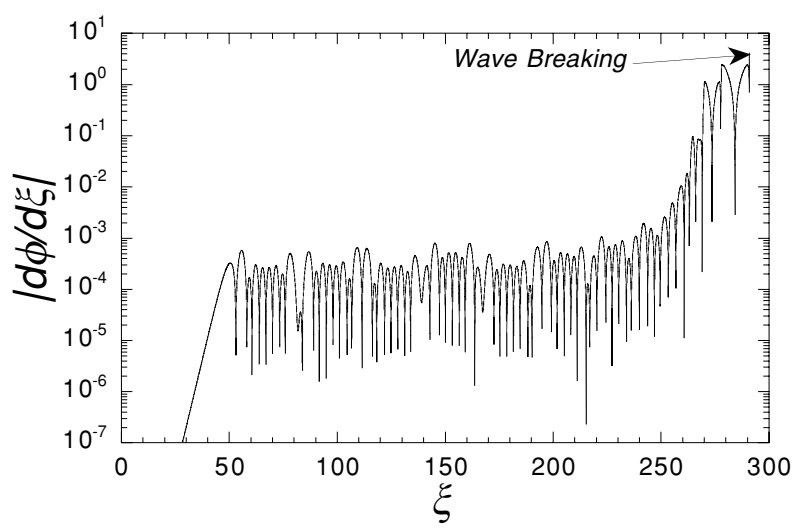

Figure 4. 'Time' series for the electric field $|d \phi / d \xi|$ for $\eta=1.0004 \eta_{*}$.

a slightly chaotic $\phi$ motion. Those cases where $\psi=0$ but $\phi \neq 0$, correspond to quasi-neutral $\psi$ pulses accompanied by trails of $\phi$ activity as described in Kuehl and Zang (1993) and Sudan et al. (1997). We see that trails can be regular or chaotic. Finally, for large enough $\eta$, KAM surfaces no longer arrest diffusion and wave breaking does occur as $r_{\mathrm{e}} \rightarrow 0$, as we have checked. At this point adiabatic motion is lost and this is likely to correspond to the point where small-amplitude solitary solutions are entirely destroyed as commented in Poornakala et al. (2002) and Farina and Bulanov (2001).

\section{Final conclusions}

To summarize, we have used the tools of nonlinear dynamics to examine the problem of wave propagation in relativistic cold plasmas, discussing underdense regimes appropriate to wakefield schemes. Nonlinear dynamics provides a unified view of the problem, thus allowing one to address several relevant questions simultaneously. In this paper we have kept our interest focused on weakly nonlinear modes where a transition from adiabatic to non-integrable dynamics was observed. Starting with very low-amplitude regimes near the onset of modulational instability, one has either isolated pulses or pulses coexisting with regular $\phi$ trails. As one increases $\eta$, thus moving away from the onset, pulses with slightly larger amplitude exist but are never fully isolated since tangent bifurcations annihilate the central fixed point and create ubiquitous chaotic electrostatic trails. However, electrostatic activity is still surrounded by KAM surfaces and therefore confined to small amplitudes. Now as one pushes amplitudes a little higher, isolating KAM surfaces are destroyed, pulses are no longer possible at all and wave breaking does occur. There are therefore three clearly identified regimes in the problem: (i) regular or adiabatic regimes where the dynamics is approximately integrable; (ii) a weakly chaotic regimes where chaos is present but chaotic diffusion is still absent due to the presence of lingering isolating KAM surfaces; and finally (iii) diffusive chaotic regimes where isolating KAM surfaces are absent. Apart from weakly nonlinear pulses, one also has the possibility of strongly nonlinear solutions (Kozlov et al. 1979; Farina and Bulanov 2001; Poornakala et al. 2002) which shall be scrutinized in future publications with the help of tools from nonlinear dynamics. 
Acknowledgement

We acknowledge partial support by CNPq, Brazil.

\section{References}

Bingham, R. 2003 Nature 424, 258.

Farina, F. and Bulanov, S. V. 2001 Phys. Rev. Lett. 86, 5289; Plasma Phys. Rep. 27, 641.

Joshi, C. and Katsouleas, T. 2003 Physics Today 56, 47.

Kozlov, V. A., Litvak, A. G. and Suvorov, E. V. 1979 Zh. Eksp. Teor. Fiz 76, 148.

Kuehl, H. H. and Zhang, C. Y. 1993 Phys. Rev. E 48, 1316.

Lichtenberg, A. J. and Lieberman, M. A. 1992 Regular and Stochastic Dynamics. New York: Springer.

Mendonça, J. T. 2001 Theory of Photon Accelerator. Bristol: IOP Publishing.

Mofiz, U. A. and de Angelis, U. 1985 J. Plasma Phys. 33, 107.

Pakter, R. and Rizzato, F. B. 2001 Phys. Rev. Lett. 87, 044801.

Poornakala, S., Das, A., Sen, A. and Kaw, P. K. 2002 Phys. Plasmas 9, 1820.

Rizzato, F. B., Lopes, S. R. and Chian, A.C.-L. 1997 Phys. Rev. E 55, 3423.

Rizzato, F. B. and Pakter, R. 2002 Phys. Rev. Lett. 89, 184102.

Shukla, P. K., Rao, N. N., Yu, M. Y. and Tsintsadze, N. L. 1986 Phys. Lett. 138, 1.

Sudan, R. N., Dimant, Y. S. and Shiryaev, O. B. 1997 Phys. Plasmas 4, 1489.

Tajima, T. and Dawson, J. M. 1979 Phys. Rev. 43, 267. 